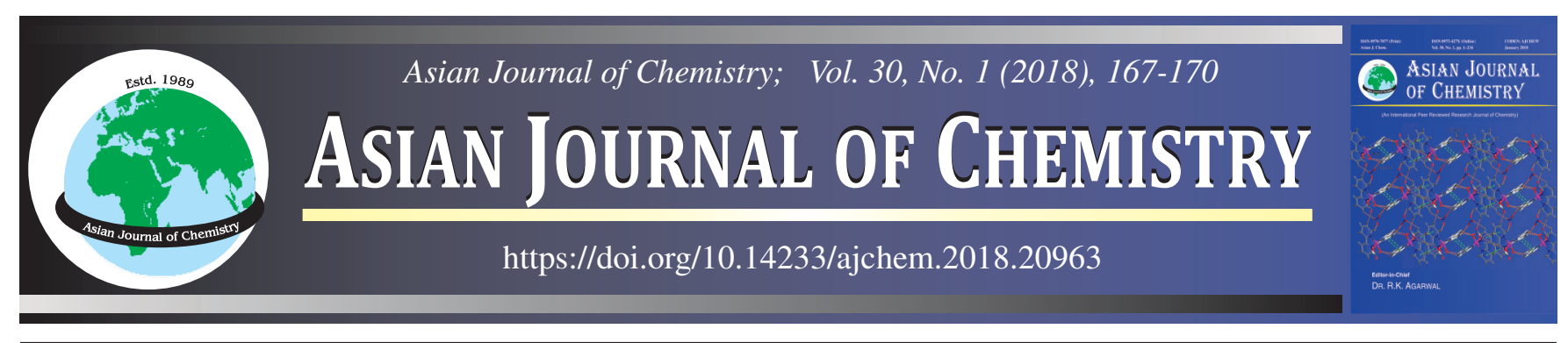

\title{
Rate Enhancements Due to Ultrasound in Isoquinolinium Dichromate and Isoquinolinium Chlorochromate Catalyzed Chlorination of Aromatic Compounds in Presence of $\mathrm{KHSO}_{4} / \mathrm{KCl}$
}

\author{
K.C. Rajanna ${ }^{1, *}$, A. Sambashiva Rao ${ }^{2,3}$, I.E. ChakraVarthi ${ }^{2}$ and K. Rajendar Reddy ${ }^{1}$
}

${ }^{1}$ Department of Chemistry, Osmania University, Hyderabad-500 007, India

${ }^{2}$ Department of Chemistry, Rayalaseema University, Kurnool-518 007, India

${ }^{3}$ Department of Chemistry, Maturi Venkata Subba Rao Engineering College, Hyderabad-500 007, India

*Corresponding author: E-mail: kcrajannaou@yahoo.com

Received: 9 August 2017;

Accepted: 31 October 2017;

Published online: 30 November 2017;

AJC-18670

Chlorination of aromatic compounds underwent magnificent rate accelerations in isoquinolinium dichromate and isoquinolinium chlorochromate catalyzed chlorination of aromatic hydrocarbons in the presence of $\mathrm{KCl}$ and $\mathrm{KHSO}_{4}$. Reaction times reduced highly significantly from $4-5 \mathrm{~h}$ in conventional protocol to 30-40 min under sonication, followed by high yields of monochloro derivatives as products with high regioselectivity.

Keywords: Chlorination, Aromatic compounds, Isoquinolinium chlorochromate, Isoquinolinium dichromate, Sonication.

ᄂ - - - - - - - - - - - - - - - - - - - - - - - - - - - - - -

\section{INTRODUCTION}

Like several other electrophilic aromatic substitution reactions, chlorination of aromatic compounds is also an evergreen and prominent synthetic protocol because of its uses in the synthesis of diversified aromatic compounds such as aromatic ethers and thioethers, amines, arylhydrazines, benzonitriles, fluoroaromatic, silylated aromatic hydrocarbons and many more functionalities [1-3]. Reagents such as molecular chlorine [4], sulfuryl chloride [5], alkyl and acyl hypochlorites [6], inorganic chlorides [7], $m$-CPBA/18-crown-6/ $\mathrm{KCl}$ [8], $\mathrm{NaBO}_{3} / \mathrm{Na}_{2} \mathrm{WO}_{4} /$ $\mathrm{KCl}$ [9], $\mathrm{H}_{2} \mathrm{O}_{2} / \mathrm{NH}_{4} \mathrm{VO}_{3} / \mathrm{KCl}$ [10], N-chlorosuccinimide [11], benzyltrimethyl ammonium tetrachloroiodate $\left(\mathrm{BTMAICl}_{4}\right)$ [12], dichlorinemonoxide [13], $\mathrm{PhICl}_{2}$ in trifluoroacetic acid [14], $\mathrm{N}$-chloroamines [15], N-chloroamides and N-chlorosulfonamides [16]. Many of the reported methods have some limitations such as use of strong and non-selective chlorinating agents, toxic and expensive reagents, low yields and long reaction times. In some protocols, after completion of the reaction, large amount of acid waste is drained through the outlets of laboratories and industries. Oxychlorination methods were among some such protocols through which one can address few issues and also achieve smooth chlorination $[17,18]$. Onium halochromates and onium dichromates were among the few most effective reagents, which could be used for oxidation as well as oxyhalogenation of organic compounds [19]. Srinivasan et al. [20] synthesiszed isoquinolinium dichromate (a new oniumdichromate) which proved as a versatile reagent for selective oxidation of primary and secondary alcohols under mild conditions. It oxidized vicinal and non-vicinal diols to the corresponding $\alpha$-hydroxy carbonyl compounds. Recently, we have accomplished the use of isoquinolinium dichromate and isoquinolinium chlorochromate as efficient catalysts to trigger oxidative bromination and iodination of aromatic hydrocarbons with $\mathrm{KBr} / \mathrm{KI}$ and $\mathrm{KHSO}_{4}$ under acidfree conditions. Reaction times reduced highly significantly under sonication, followed by corresponding monobromo derivatives in very good yield with high regioselectivity [21]. Encouraged by this protocol we have embarked on exploring the possibility of isoquinolinium chlorochromate (IQCC) and isoquinolinium dichromate (IQDC) as catalysts to achieve oxidative chlorination of aromatic compounds with various functional groups such as phenols, anilines and acetanilides. We also wish to explore the use of ultrasound to assist IQCC and IQDC mediated chlorination reactions with a view to accelerate chemical reactions for achieving better yields as well as improving the greenery of reaction protocols. Earlier reviews and publications in this field [22-32] proved the importance of ultrasonically assisted organic synthesis and also highlighted that sonication using ultrasound is not only simple, but also satisfies both economical and environmental demands, as recommended by Anastas and Warner in the green chemistry formulations [33].

\section{EXPERIMENTAL}

All chemicals used were of synthesis grade reagents and procured from Merck, S.D. Fine or Avra Chemicals. Isoquino- 
linium dichromate (IQDC) and isoquinolinium chlorochromate (IQCC) were prepared as reported method [20,21].

General procedure for conventional chlorination reactions: Organic compound $(10 \mathrm{mmol})$ potassium chloride $(\mathrm{KCl} 10$ $\mathrm{mmol}$ ) and (1 mmol) $\mathrm{Cr}(\mathrm{VI})$ reagent (IQCC or IQDC) and solvent (aq. acetontrile or DCE) were taken in a clean round bottom flask. About $50 \mathrm{mg}$ of $\mathrm{KHSO}_{4}$ is also added to the contents of reaction flask and the reaction mixture is refluxed for about $4-5 \mathrm{~h}$ at $50-60{ }^{\circ} \mathrm{C}$ till the completion reaction, as ascertained by thin layer chromatography (TLC). The reaction mixture is then treated with 5\% sodium thiosulphate solution followed by the addition of ether. The aqueous layer was separated from the ether layer. The ether is evapourated from organic layer under vacuum, and purified with column chromatography using chloroform: $n$-hexane (9:1) as eluent to get pure chlorinated aromatic compound as product $[2,3]$.

General procedure for ultrasonically assisted chlorination reactions: The general procedure for ultrasonically assisted chlorination reaction is largely similar to the preceeding conventional method. After mixing the reactants in a clean round bottom flask, is placed in an ultrasound assited sonicator and sonicated at room temperature till the reaction is completed (about 30$40 \mathrm{~min}$ ). Progress of the reaction was monitored by TLC technique. Work up procedure after completion of the reaction mixture is similar to as described above.

\section{RESULTS AND DISCUSSION}

The chlorination reactions of aromatic compounds were conducted using isoquinolinium dichromate (IQDC)/ $\mathrm{KCl}$ and isoquinolinium chlorochromate (IQCC) $/ \mathrm{KCl}$ combinations in aqueous $\mathrm{KHSO}_{4}$ under mineral acid free conditions. Described methods worked out well for an array of functionalities such as phenols, anilines or acetanilides (Scheme-I).

Each reaction is repeated at least half a dozen times and the reaction times are reproducible with an accuracy of $\pm 5 \mathrm{~min}$. The chlorination of aromatic compounds required 4-5 $\mathrm{h}$ under conventional conditions at reflux temperatures, but under soni-

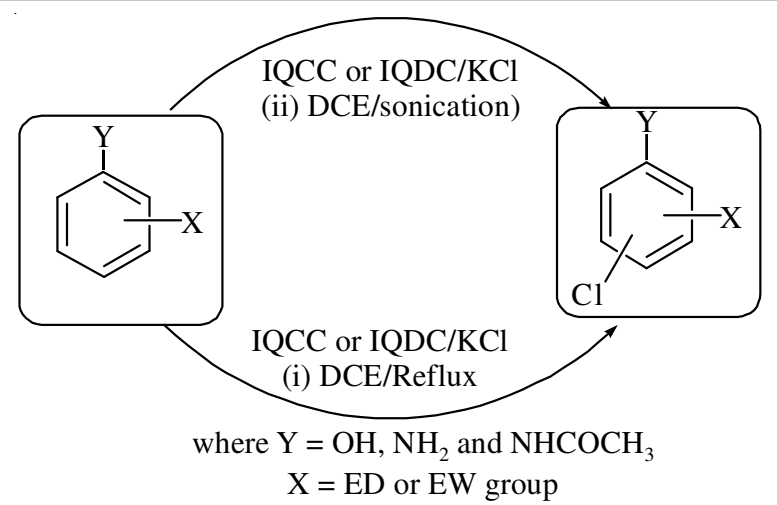

Scheme-I: IQDC and IQCC triggered chlorination of aromatic compounds

cation the reaction times are drastically reduced to about 30 to $40 \mathrm{~min}$ followed by considerable yield enhancements. The products of these reactions were characterized by spectroscopic and physical data with authentic samples and found to be satisfactory with literature reports [2,3]. The yields of major products are given in Table-1.

\section{Spectroscopic data of some synthesized compounds:}

4-Chlorophenol: ${ }^{1} \mathrm{H}$ NMR (400 MHz, $\left.\mathrm{CDCl}_{3}\right) \delta$ 7.247.17 (m, 2H, Ph), 6.77-6.74 (m, 2H, Ph), 5.27 (s, 1H, OH); HRMS (ESI-TOF) $m / z$ : calcd. for $\mathrm{C}_{6} \mathrm{H}_{5} \mathrm{OCl} 127.0029$, found 126.9956.

2-Methoxy-4-chlorophenol: ${ }^{1} \mathrm{H}$ NMR (400 MHz, DMSO$\left.d_{6}\right) \delta 9.20(\mathrm{~s}, 1 \mathrm{H}, \mathrm{OH}), 6.95(\mathrm{~d}, J=2.0 \mathrm{~Hz}, 1 \mathrm{H}, \mathrm{Ph}), 6.79-6.75$ (m, 2H, Ph), 3.78 (s, 3H, $\mathrm{OCH}_{3}$ ); HRMS (ESI-TOF) $\mathrm{m} / \mathrm{z}$ : calcd. for $\mathrm{C}_{7} \mathrm{H}_{6} \mathrm{O}_{2} \mathrm{Cl}$ 157.0135, found 157.0056.

2-Chloro-4-methoxyphenol: ${ }^{1} \mathrm{H}$ NMR (400 MHz, DMSO$\left.d_{6}\right) \delta 9.55(\mathrm{~s}, 1 \mathrm{H}, \mathrm{OH}), 6.94-6.90(\mathrm{~m}, 2 \mathrm{H}, \mathrm{Ph}), 6.76(\mathrm{dd}, J=$ 3.2, $8.8 \mathrm{~Hz}, 1 \mathrm{H}, \mathrm{Ph}), 3.69$ (s, 3H, $\mathrm{OCH}_{3}$ ); HRMS (ESI-TOF) $\mathrm{m} / \mathrm{z}$ : calcd. for $\mathrm{C}_{7} \mathrm{H}_{6} \mathrm{ClO}_{2}$ 157.0135, found 157.0059.

4-Chloro-3-methylphenol: ${ }^{1} \mathrm{H}$ NMR $(400 \mathrm{MHz}$, DMSO$\left.d_{6}\right) \delta 9.51(\mathrm{~s}, 1 \mathrm{H}, \mathrm{OH}), 7.16(\mathrm{~d}, J=2.4 \mathrm{~Hz}, 1 \mathrm{H}, \mathrm{Ph}), 6.74(\mathrm{~d}, \mathrm{~Hz}$, 1H, Ph), 6.62 (dd, , $8.8 \mathrm{~Hz}, 1 \mathrm{H}, \mathrm{Ph}), 2.24$ (s, 3H, $\mathrm{CH}_{3}$ ); HRMS (ESI-TOF) $m / z$ : calcd. for $\mathrm{C}_{7} \mathrm{H}_{6} \mathrm{ClO} 141.0185$, found 141.0116 .

\begin{tabular}{|c|c|c|c|c|c|c|}
\hline \multirow{3}{*}{ S. No. } & \multicolumn{4}{|c|}{$\begin{array}{c}\text { TABLE-1 } \\
\text { OXIDATIVE CHLORINATION OF AROMATIC HYDROCARBONS } \\
\text { Reaction time Conventional: } 4-5 \text { h; Sonication: } 30-40 \text { min }\end{array}$} & & \\
\hline & \multirow{2}{*}{ Substrate } & \multirow{2}{*}{ Product } & \multicolumn{2}{|c|}{ Conventional yield (\%) } & \multicolumn{2}{|c|}{ Sonication yield (\%) } \\
\hline & & & $\mathrm{KCl} / \mathrm{IQCC}$ & $\mathrm{KCl} / \mathrm{IQDC}$ & $\mathrm{KCl} / \mathrm{IQCC}$ & $\mathrm{KCl} / \mathrm{IQDC}$ \\
\hline 1 & Phenol & 4-Chloro Phenol & 70 & 68 & 71 & 70 \\
\hline 2 & $o$-Cresol & 4-Chloro-2-methylphenol & 65 & 60 & 66 & 62 \\
\hline 4 & $m$-Cresol & 4-chloro-3-methylphenol & 66 & 70 & 68 & 71 \\
\hline 5 & 4-Chloro phenol & 2,4-Dichloro phenol & 55 & 60 & 59 & 63 \\
\hline 6 & 2-Chloro phenol & 2,4-Dichloro phenol & 59 & 61 & 62 & 64 \\
\hline 7 & 4-Bromo phenol & 4-Bromo-2-chloro phenol & 60 & 66 & 66 & 69 \\
\hline 8 & 1,4-Dihydroxy benzene & 2-Chloro benzene-1,4-diol & 71 & 74 & 76 & 72 \\
\hline 9 & 1-Naphthol & 2-Chloro-1-naphthol & 69 & 73 & 68 & 71 \\
\hline 10 & Benzaldehyde & 3-Chloro benzaldehyde & 70 & 73 & 71 & 73 \\
\hline 12 & 4- $\mathrm{NO}_{2}$ phenol & 4- $\mathrm{NO}_{2}$-2-chloro phenol & 65 & 69 & 68 & 70 \\
\hline 13 & 2-OH benzaldehyde & 5-Chloro-2-OH-benzaldehyde & 55 & 59 & 59 & 60 \\
\hline 14 & 4-OH benzaldehyde & 3-Chloro-4-OH-benzaldehyde & 65 & 69 & 69 & 70 \\
\hline 15 & Toluene & 2-Chlorotoluene & 69 & 70 & 68 & 69 \\
\hline 16 & 2-OH-benzoic acid & 3-Chloro-6-OH- benzoic acid & 69 & 70 & 71 & 70 \\
\hline 17 & 2-OH-aniline & 3-Chloro-4-OH-aniline & 66 & 68 & 70 & 77 \\
\hline 18 & 4-Cl-acetophenone & 3,4-di-Cl-acetophenone & 71 & 67 & 69 & 70 \\
\hline
\end{tabular}


3-Chloro-5-hydroxy benzaldehyde: ${ }^{1} \mathrm{H}$ NMR $(400 \mathrm{MHz}$, DMSO- $\left.d_{6}\right) \delta 10.93(\mathrm{~s}, 1 \mathrm{H}), 10.23(\mathrm{~s}, 1 \mathrm{H}), 7.60(\mathrm{~d}, \mathrm{~Hz}, 1 \mathrm{H}$, $\mathrm{Ph}), 7.54(\mathrm{dd},, 8.8 \mathrm{~Hz}, 1 \mathrm{H}, \mathrm{Ph}), 7.05(\mathrm{~d}, \mathrm{~Hz}, 1 \mathrm{H}, \mathrm{Ph}) ; \mathrm{HRMS}$ (ESI-TOF) $m / z$ : calcd. for $\mathrm{C}_{7} \mathrm{H}_{4} \mathrm{ClO}_{2} 154.9978$, found 154.9898 .

4-Chloro-2-methylphenol: ${ }^{1} \mathrm{H}$ NMR $(400 \mathrm{MHz}, \mathrm{DMSO}-$ $\left.d_{6}\right) \delta 9.52(\mathrm{~s}, 1 \mathrm{H}, \mathrm{OH}), 7.10(\mathrm{~d}, \mathrm{~Hz}, 1 \mathrm{H}, \mathrm{Ph}), 7.01(\mathrm{dd},, 8.4 \mathrm{~Hz}$, $1 \mathrm{H}, \mathrm{Ph}), 6.78(\mathrm{~d}, \mathrm{~Hz}, 1 \mathrm{H}, \mathrm{Ph}), 2.11\left(\mathrm{~s}, 3 \mathrm{H}, \mathrm{CH}_{3}\right)$; HRMS (ESITOF) $\mathrm{m} / z$ : calcd. for $\mathrm{C}_{7} \mathrm{H}_{6} \mathrm{OCl} 141.0185$, found 141.0107 .

4-Chloro-2-nitroaniline: Yellow orange powder; ${ }^{1} \mathrm{H}$ NMR (400 MHz, DMSO) $\delta 7.90(\mathrm{~d}, J=2.42,1 \mathrm{H}, \mathrm{Ar}), \delta 7.28(\mathrm{dd}$, $J=9.20,2.24,1 \mathrm{H}, \mathrm{Ar}), \delta 7.06$ (d, $J=9.22,1 \mathrm{H}, \mathrm{Ar}) \delta 7.55$ (bs, $1 \mathrm{H}, \mathrm{NH}_{2}$ ) ppm; MS (APCI): calcd. for $\mathrm{C}_{6} \mathrm{H}_{5} \mathrm{~N}_{2} \mathrm{O}_{2} \mathrm{Cl}[\mathrm{M}]^{+}$ 172.57 , found $172[\mathrm{M}]^{+}$.

3-Chloro-4-hydroxybenzaldehyde: Light brown powder; ${ }^{1} \mathrm{H}$ NMR (400 MHz, DMSO) $\delta 9.78$ (s, 1H, CHO), $\delta 7.81$ (d, $J=1.80 \mathrm{~Hz}, 1 \mathrm{H}, \mathrm{Ar}), \delta 7.64(\mathrm{dd}, J=8.40,1.80 \mathrm{~Hz}, 1 \mathrm{H}, \mathrm{Ar})$, $\delta 7.12(\mathrm{~d}, J=8.32 \mathrm{~Hz}, 1 \mathrm{H}, \mathrm{Ar}) \mathrm{ppm} ; \mathrm{MS}$ : calcd. For $\mathrm{C}_{7} \mathrm{H}_{5} \mathrm{O}_{2} \mathrm{Cl}$ $[\mathrm{M}]^{+} 156,5$, found $155[\mathrm{M}-1]^{+}$.

5-Chlorosalicylic acid: White crystals; ${ }^{1} \mathrm{H}$ NMR $(400$ MHz, DMSO) $\delta 9.05(\mathrm{~s}, 1 \mathrm{H}, \mathrm{OH}), \delta 7.79(\mathrm{~d}, J=2.52 \mathrm{~Hz}, 1 \mathrm{H}$, $\mathrm{Ar}$ ), $\delta 7.38$ (dd, $J=8.80,2.52 \mathrm{~Hz}, 1 \mathrm{H}, \mathrm{Ar}), \delta 6.91$ (d, $J=8.88$ $\mathrm{Hz}, 1 \mathrm{H}, \mathrm{Ar}$ ) ppm; MS: calcd. for $\mathrm{C}_{7} \mathrm{H}_{5} \mathrm{O}_{3} \mathrm{Cl}[\mathrm{M}]+172$, found 171.

We have also tried to to explain the mechanism of IQDC/ $\mathrm{KCl}$ mediated chlorination reaction. It is well established in earlier reports that in aquoues acidic media $\mathrm{K}_{2} \mathrm{Cr}_{2} \mathrm{O}_{7}$ or $\mathrm{Cr}(\mathrm{VI})$ exists in several reactive forms, such as $\mathrm{HCrO}_{4}^{-}, \mathrm{H}_{2} \mathrm{CrO}_{4},\left[\mathrm{HCrO}_{3}\right]^{+}$ and $\mathrm{HCrO}_{3} \mathrm{~B}$ (where $\mathrm{B}=\mathrm{HSO}_{4}^{-}, \mathrm{ClO}_{4}^{-}$or $\mathrm{Cl}^{-}$) $[34,35]$. On the basis of fact that quinolinium dichromate (QDC) is related to $\mathrm{K}_{2} \mathrm{Cr}_{2} \mathrm{O}_{7}$, we have formulated similar types of reactive species with quinolinium ion background [36]. Further, it is also of interest to note that isoquinolinium dichromate (IQDC) resembles the structure of quinolinium dichromate (QDC), following species could be considered according to the following equilibria:

$$
(\mathrm{IQH})_{2} \mathrm{Cr}_{2} \mathrm{O}_{7}+\mathrm{H}_{2} \mathrm{O} \rightleftharpoons 2\left[(\mathrm{IQH})+\left(\mathrm{HCrO}_{4}\right)^{-}\right]
$$

As the reactions are conducted in presence of aqueous $\mathrm{KHSO}_{4}$ medium, it is more likely that the bisulfate ion dissociates to give $\left(\mathrm{H}^{+}\right)$creating mild acid reaction conditions. Protons $\left(\mathrm{H}^{+}\right)$ thus released could be used to protonate IQDC, leading to the formation of active $\left[(\mathrm{IQH})^{+}\left(\mathrm{HCrO}_{4}\right)^{-}\right]$(IQ bound chromic acid) species.

$$
\mathrm{HSO}_{4} \rightleftharpoons \mathrm{SO}_{4}^{2-}+\mathrm{H}^{+}
$$

$$
(\mathrm{IQH})^{+}\left(\mathrm{HCr}_{2} \mathrm{O}_{4}\right)^{-}+\mathrm{H}^{+} \rightleftharpoons\left[(\mathrm{IQH}) \mathrm{CrO}_{3}\right]^{+}+\mathrm{H}_{2} \mathrm{O}
$$

Active species thus formed may further react with chloride ion to afford $\left[(\mathrm{IQH}) \mathrm{CrO}_{3} \mathrm{Cl}\right]$ species, since the reactions are conducted in excess $\mathrm{KCl}$.

$$
\left[(\mathrm{IQH}) \mathrm{CrO}_{3}\right)^{+}+\mathrm{Cl}^{-} \rightleftharpoons\left[(\mathrm{IQH}) \mathrm{OCrO}_{2} \mathrm{Cl}\right]
$$

Finally, aromatic substrates undergo mono chlorination through the formation of cyclic intermediate with aromatic compound as shown in Scheme-II.

On the other hand, the most plausible mechanism in IQCC triggered reactions could be proposed by formulating isoquinolinium chlorochromate (IQCC) in the lines of other onium halochromate species [37-40]. Since the reactions are studied in aqueous $\mathrm{KHSO}_{4}$ medium, IQCC could exists as $\left(\mathrm{IQHOCrO}_{2} \mathrm{Cl}\right)$

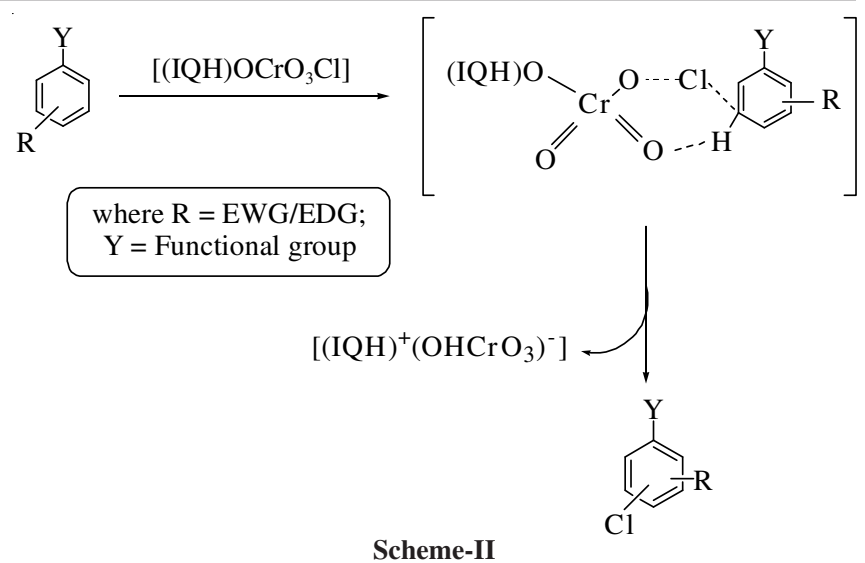

and its protonated form $(\mathrm{IQHOCr}(\mathrm{OH}) \mathrm{Cl})^{+}$, by capturing $\mathrm{H}^{+}$ from the dissociated bisulfate species, according to the following equilibria:

$$
(\mathrm{IQHOCrO} 2 \mathrm{Cl})+\mathrm{H}^{+} \rightleftharpoons(\mathrm{IQHOCr}(\mathrm{OH}) \mathrm{OCl})^{+}
$$

The protonated chromium(VI) species thus formed $(\mathrm{IQHOCr}(\mathrm{OH}) \mathrm{OCl})^{+}$being a stronger electrophile, may further react with chloride ion to afford $[\mathrm{IQHOCr}(\mathrm{OH})(\mathrm{OCl}) \mathrm{Cl}]$ species, since the reactions are conducted in excess $\mathrm{KCl}$.

\section{$[\mathrm{IQHOCr}(\mathrm{OH}) \mathrm{OCl}]^{+}+\mathrm{Cl}^{-} \rightleftharpoons[\mathrm{IQHOCr}(\mathrm{OH})(\mathrm{OCl}) \mathrm{Cl}](6)$}

Finally, aromatic substrates undergo mono chlorination through the attack of $\mathrm{Cl}^{+}$of $[\mathrm{IQHOCr}(\mathrm{OH})(\mathrm{OCl}) \mathrm{Cl}]$ on the aromatic ring as shown Scheme-III.

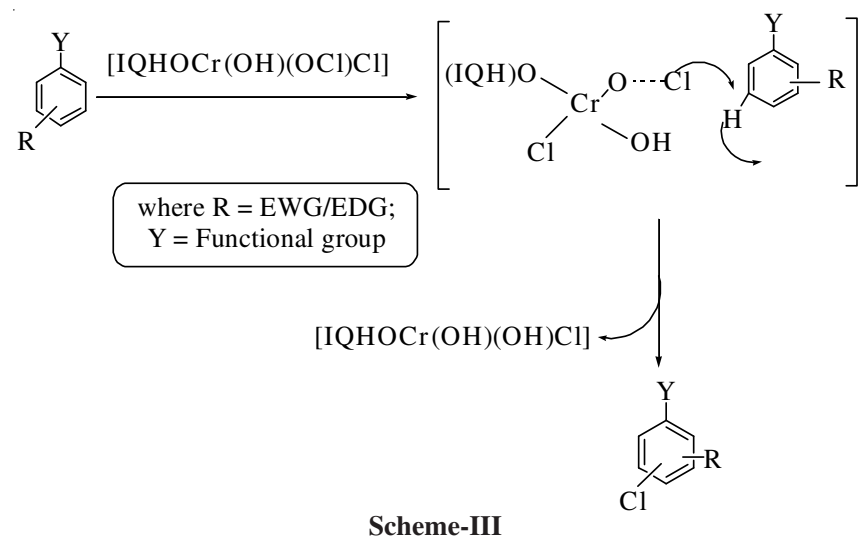

The observed rate enhancements in ultrasonically assisted reactions could be explained due to cavitation, a physical process that creates, enlarges implodes gaseous and vaporous cavities in an ultrasonically assisted (irradiated) liquid. Cavitation induces very high local temperatures in the reaction mixture and enhances mass transfer [20-29].

\section{Conclusion}

In summary, isoquinolinium chlorochromate (IQCC) and isoquinolinium dichromate (IQDC) as cost-effective catalysts to trigger chlorination of aromatic compounds in presence of $\mathrm{KCl}$ and small amount of $\mathrm{KHSO}_{4}$ is successfully explored. The use of mineral acid is completely avoided in these protocols. Reaction times drastically reduced from $4-5 \mathrm{~h}$ (in conventional) to 30-40 min under sonication. The reactions occurred under mild and under environmentally safe conditions with simple 
work up at room temperature. Thus, it is believed that the developed protocols are one of the important contributions in the area of chlorination reactions.

\section{REFERENCES}

1. (a) P.B.D. De la Mare, Electrophilic Halogenation, Cambridge University Press: Cambridge (1976).

(b) R. Taylor, Electrophilic Aromatic Substitution, Wiley: Chichester, pp. 362 (1990).

(c) Ullmann's Encyclopedia of Industrial Chemistry, Wiley-VCH, Weinheim, edn 6 (1998).

2. (a) N. Narender, P. Srinivasu, S.J. Kulkarni and K.V. Raghavan, Synth. Commun., 32, 279 (2002); https://doi.org/10.1081/SCC-120002013.

(b) N. Narender, K.V.V. Krishna Mohan, P. Srinivasu, S.J. Kulkarni and K.V. Raghavan, Indian J. Chem., 43B, 1335 (2004)

3. (a) H.A. Muathen, Monatsh. Chem., 130, 1493 (1999); https://doi.org/10.1007/s007060050309.

(b) Y. Xiong, H. Duan, X. Meng, Z. Ding and W. Feng, J. Chem., Article ID 2960414 (2016);

https://doi.org/10.1155/2016/2960414.

(c) K.H. Chung, H.J. Kim, H.R. Kim, and E.K. Ryu, Synth. Commun., 20, 2991 (1990):

https://doi.org/10.1080/00397919008051517.

4. (a) R.H. Huston and A.H. Neeley, J. Am. Chem. Soc., 57, 2176 (1935); https://doi.org/10.1021/ja01314a040.

(b) D.R. Harvey and R.O.C. Norman, J. Chem. Soc., 3604 (1961); https://doi.org/10.1039/jr9610003604.

5. (a) M. Hojo and R. Masuda, Synth. Commun., 5, 169 (1975); https://doi.org/10.1080/00397917508064104.

(b) L. Delaude and P. Laszlo, J. Org. Chem., 55, 5260 (1990); https://doi.org/10.1021/jo00305a023.

6. (a) M. Anbar and D. Ginsburg, Chem. Rev., 54, 925 (1954); https://doi.org/10.1021/cr60172a002.

(b) D. Ginsburg, J. Am. Chem. Soc., 73, 2723 (1951); https://doi.org/10.1021/ja01150a084.

(c) K. Smith, M. Butters, W.E. Paget and B. Nay, Synthesis, 1155 (1985); https://doi.org/10.1055/s-1985-31461.

(d) I. Lengyel, V. Cesare and R. Stephani, Synth. Commun., 28, 1891 (1998);

https://doi.org/10.1080/00397919808007021.

(e) K. Smith, M. Butters and B. Nay, Synthesis, 1157 (1985);

https://doi.org/10.1055/s-1985-31462.

7. (a) D. Masilamani and M.M. Rogic, J. Org. Chem., 46, 4486 (1981); https://doi.org/10.1021/jo00335a033.

(b) M. Srebnik, R. Mechoulam and I. Yona, J. Chem. Soc., Perkin Trans. I, 1423 (1987);

https://doi.org/10.1039/p19870001423

(c) E.M. Kosower, W.J. Cole, G.-S. Wu, D.E. Cardy and G.J. Meisters, J. Org. Chem., 28, 630 (1963);

https://doi.org/10.1021/jo01038a007.

(d) H. Lübbecke and P. Boldt, Tetrahedron, 34, 1577 (1978);

https://doi.org/10.1016/0040-4020(78)80185-9.

8. N. Usami, K. Kobana, H. Yoshida, T. Kimura, K. Watanabe, H. Yoshimura and I. Yamamoto, Chem. Pharm. Bull. (Tokyo), 46, 1462 (1998); https://doi.org/10.1248/cpb.46.1462.

9. B.P. Bandgar and N.J. Nigal, Synth. Commun., 28, 3225 (1998); https://doi.org/10.1080/00397919808004426.

10. V.R. Hegde, G.C.G. Pais, R. Kumar, P. Kumar and B. Pandey, J. Chem. Res. (S), 62 (1996)

11. (a) Y. Goldberg and H. Alper, J. Mol. Catal., 88, 377 (1994); https://doi.org/10.1016/0304-5102(93)E0278-O.

(b) F.L. Lambert, W.D. Ellis and R.J. Parry, J. Org. Chem., 30, 304 (1965); https://doi.org/10.1021/jo01012a512.

12. S. Kajigaeshi, Y. Shinmasu, S. Fujisaki and T. Kakinami, Bull. Chem. Soc. Jpn., 63, 941 (1990);

https://doi.org/10.1246/bcsj.63.941.

13. F.D. Marsh, W.B. Farnham, D.J. Sam and B.E. Smart, J. Am. Chem. Soc., 104, 4680 (1982); https://doi.org/10.1021/ja00381a032.

14. L.J. Andrews and R.M. Keefer, J. Am. Chem. Soc., 82, 5823 (1960); https://doi.org/10.1021/ja01507a014.
15. (a) J.R. Lindsay Smith, L.C. McKeer and J.M. Taylor, J. Chem. Soc., Perkin Trans. 2, 1529 (1989);

https://doi.org/10.1039/P29890001529.

(b) J.R. Lindsay Smith, L.C. McKeer and J.M. Taylor, J. Chem. Soc., Perkin Trans. 2, 385 (1988); https://doi.org/10.1039/P29880000385.

(c) J. R. Lindsay Smith, L.C. McKeer, and J.M. Taylor, J. Chem. Soc., Perkin Trans. 2, 1537 (1989); https://doi.org/10.1039/P29890001537.

16. P. Bovonsombat and E. Mcnelis, Synthesis, 237 (1993); https://doi.org/10.1055/s-1993-25839.

17. (a) E.B. Merkushev, Synthesis, 923 (1988); https://doi.org/10.1055/s-1988-27758.

(b) J.K. Stille, Angew. Chem. Int. Ed. Engl., 25, 508 (1986); https://doi.org/10.1002/anie.198605081. (c) A. Suzuki, Pure Appl. Chem., 63, 419 (1991); https://doi.org/10.1351/pac199163030419.

18. (a) A. Bachki, F. Foubelo and M. Yus, Tetrahedron, 50, 5139 (1994); https://doi.org/10.1016/S0040-4020(01)90424-7.

(b) G.A. Olah, Q. Wang, G. Sandford and G.K. Surya Prakash, J. Org. Chem., 58, 3194 (1993); https://doi.org/10.1021/jo00063a052.

(c) B. Akhlaghinia and M. Rahmani, Turk. J. Chem., 33, 67 (2009).

(d) J. March, Advanced Organic Chemistry, Wiley-Interscience, New York, edn 4 (2000).

19. (a) S. Patel and B.K. Mishra, Tetrahedron, 63, 4367 (2007); https://doi.org/10.1016/i.tet.2007.02.073.

(b) B. Tamami and A.R. Kiasat, Iran. Polym. J., 6, 273 (1997). (c) A.F. Luzzio and F.S. Guziec Jr., Org. Prep. Proced. Int., 20, 533 (1988); https://doi.org/10.1080/00304948809356301.

20. R. Srinivasan, S. Akila, J. Caroline and K. Balasubramanian, Synth. Commun., 28, 2245 (1998); https://doi.org/10.1080/00397919808007040.

21. A.S. Rao, K.C. Rajanna, K.R. Reddy and S. Kulkarni, Synth. React. Inorg. Met.-Org. Nano-Met. Chem., 46, 832 (2016); https://doi.org/10.1080/15533174.2014.989596.

22. S.V. Ley and C.M.R. Low, Ultrasound in Synthesis, Springer, Berlin, (1989).

23. W.T. Richards and A.L. Loomis, J. Am. Chem. Soc., 49, 3086 (1927); https://doi.org/10.1021/ja01411a015.

24. T.J. Mason and J.P. Lorimer, Sonochemistry: Theory, Applications and Uses of Ultrasound in Chemistry, John Wiley \& Sons, New York (1988).

25. T.J. Mason, Chem. Soc. Rev., 26, 443 (1997); https://doi.org/10.1039/cs9972600443.

26. K.S. Suslick, Ultrasound: Its Chemical, Physical and Biological Effects, VCH, New York (1988)

27. V. Singh, K.P. Kaur, A. Khurana and G.L. Kad, Resonance, 3, 56 (1998); https://doi.org/10.1007/BF02836081.

28. T.J. Mason, Chemistry with Ultrasound, Elsevier Science Publishers Ltd., London (1990).

29. T.J. Mason and D. Peters, Practical Sonochemistry: Power Ultrasound Uses and Applications, Hoorwood Publishing, Chichester, edn 2 (2003).

30. M.A. Margulis, Advances in Sonochemistry, JAI Press, London, vol. 1, p. 49 (1990).

31. H. Fillion and J.L. Luche, ed.: J.L. Luche, Selected Experiments, In: Synthetic Organic Sonochemistry, Plenum, New York, Chap. 9 (1998).

32. R. Cella and H.A. Stefani, Tetrahedron, 65, 2619 (2009); https://doi.org/10.1016/j.tet.2008.12.027.

33. P.T. Anastas and J.C. Warner, Green Chemistry: Theory and Practice Oxford University Press; New York (1998).

34. K.B. Wiberg, Oxidations in Organic Chemistry, Part A, Academic Press, New York (1965)

35. D. Benson, Mechanisms of Oxidation by Metal Ions, Elsevier, New York (1976).

36. J.N. Reddy, S. Giridhar, K.C. Rajanna and P.K. Saiprakash, Transition Met. Chem., 21, 105 (1996); https://doi.org/10.1007/BF00136537.

37. D. Sharma, P. Pancharia, K. Vadera and P.K. Sharma, J. Sulfur Chem., 32, 315 (2011); https://doi.org/10.1080/17415993.2011.587519.

38. D. Sharma, P. Panchariya, P. Purohit and P.K. Sharma, Oxid. Commun., 35, 821 (2012).

39. S. Panwar, S. Pohani, P. Swami, S. Vyas and P.K. Sharma, Eur. Chem. Bull., 2, 904 (2013).

40. L. Mathur and A. Choudhary, Asian J. Chem., 26, 2597 (2014); https://doi.org/10.14233/ajchem.2014.15753. 